\title{
Um Estudo Experimental sobre a Luz Negra com Smartphone
}

\author{
A experimental study about the black light with smartphone
}

\author{
$\operatorname{Diogo}_{\text {Soga }}^{*}\left[0\right.$, Michele H. Ueno-Guimarães ${ }^{2}$, Mikiya Muramatsu ${ }^{1}$ \\ ${ }^{1}$ Universidade de São Paulo, Instituto de Física da USP, São Paulo \\ ${ }^{2}$ Universidade Federal de Ouro Preto, Instituto de Ciências Exatas e Biológicas, Departamento de Física, Ouro Preto, MG,
} Brasil

Recebido em 07 de Maio 2019. Revisado 10 de Setembro 2019. Aceito 05 de Novembro de 2019.

\begin{abstract}
Neste trabalho, apresentamos um estudo sobre a proposta de luz negra, usando a lanterna de um smartphone em conjunto com um filtro azul, e este conjunto emitiria luz ultravioleta, para realizar experimentos de fluorescência. Os trabalhos publicados com essa proposta não fizeram a análise do espectro de emissão dessa fonte de luz. Utilizando um espectrofotômetro, investigamos a emissão espectral da lanterna de três smartphones diferentes. Também utilizamos alguns filtros para analisar as fontes de luz citadas anteriormente. Realizamos alguns experimentos de fluorescência com uma lanterna de um smartphone e uma lâmpada de luz negra, e comparamos os resultados obtidos.
\end{abstract}

Palavras-chave: Óptica, fluorescência, luz ultravioleta, luz negra, smartphone.

This work presents a study about the proposal of the black light using a flashlight of a smartphone with a blue filter, and this set could emitted ultraviolet light, to do experiments of fluorescence. The published works with this porposal did not analyse the emission spectra of light source. Using a spectrophotometer we investigated the emission spectra of the flashlight of three different smartphones. We also used some filters to analyse the light sources cited before. We did experiments of fluorescence with the flashligth of a smartphone and a lamp of dark light, and we compared the obtained results.

Keywords: Optics, fluorescence, ultraviolet ligth, black light, smartphone.

\section{Introdução}

A fluorescência [1] é um processo físico, onde um material, ao ser iluminado por fótons de uma frequência $\nu_{1}$, ele emite fótons de frequência menor $\nu_{2}$ (ou comprimento de onda maior [1]). Por exemplo, se um material for iluminado por luz ultravioleta (UV), da ordem de 380 $\mathrm{nm}$, ele poderia emitir fótons na cor verde, da ordem de $550 \mathrm{~nm}$. Neste caso, o olho humano 11 não veria a luz UV, porque está fora da região da luz visível, mas veria a luz de cor verde. Vários materiais apresentam esta característica [2 3], por exemplo: cédulas de papel moeda, tintas de canetas marca-texto, clorofila e a carapaça do escorpião. Experimentos educativos de demonstração do fenômeno, utilizam uma fonte de luz UV, iluminando tais materiais em um local escuro e os materiais fluorescem, emitindo luz na região do visível.

Segundo Almeida et al [4], a instrumentalização na área de Ensino auxilia o educador a trazer a realidade mais perto do aluno, isto é, quando os alunos realizam experimentos com a visualização dos fenômenos físicos e de suas aplicações. No caso citado acima, a fonte de luz UV é usada para mostrar a fluorescência de alguns objetos do cotidiano.

Porém a execução de experimentos deve ser feita de maneira adequada e evitar equívocos que levam a conclusões inadequadas. Geralmente são usadas lâmpadas de luz negra e laseres que emitem UV. Alguns trabalhos publicados na Internet [5-7] propõem usar a lanterna de um smartphone, com um filtro azul como uma fonte de luz ultravioleta. Estes trabalhos não fizeram o estudo do espectro de emissão da luz emitida, aparentemente eles pressupõe que a fonte de luz proposta emita luz ultravioleta. A lanterna é formada por um ou dois LEDs de luz branca, que é utilizada para iluminar um objeto ou pessoa a ser fotografada pela câmera do smartphone. Então surgiu a dúvida, será que a lanterna realmente emite luz UV? Isto é preocupante, pois ao fotografar uma pessoa, tal radiação poderá incidir nos olhos da pessoa e/ou sobre a pele, e pode causar danos à pessoa. Neste trabalho, apresentamos um estudo sobre essa proposta para determinar se há emissão de luz UV.

*Endereço de correspondência: diogosp@usp.br 


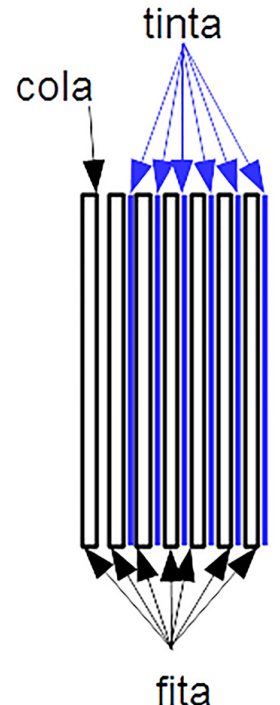

(a) Esquema da construção do filtro azul.

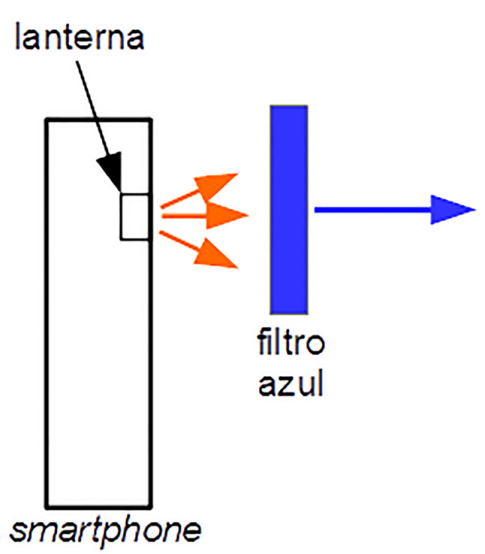

(b) A lanterna com o filtro azul.

Figura 1: Proposta de luz negra com a lanterna de smartphone.

\section{Usando a Lanterna do smartphone}

A proposta dos trabalhos da Internet [5, 7. consiste em cobrir a lanterna de um smartphone, com camadas de fita adesiva transparente. Cada camada deve ser pintada com uma caneta marcadora de CD/DVD da cor azul. O objetivo é barrar parte da luz branca da lanterna e deixar passar a luz azul e a luz UV. Assim, ao iluminar alguns objetos com esta luz, eles podem apresentar a fluorescência.

Neste estudo, o filtro azul (Fig. 1a) foi construído em separado, isto é, não foi colado ao smartphone, deste modo, o mesmo filtro pode ser usado em smartphones diferentes. Outra diferença é que foram pegos dois pedaços da fita adesiva e depois foram colados com a parte aderente entre eles, assim o lado com a cola, da fita adesiva, não fica exposta. A seguir um dos lados foi pintado com uma caneta marcadora azul. Sobre esta camada pintada foram acrescentadas outras cinco camadas de fita adesiva e tinta.

Na Figura 1b vemos o modo de operação. A luz da lanterna do smartphone passa pelo filtro azul, o que resulta em um feixe de luz com a luz UV. Como o filtro azul é um elemento passivo, isto é, não produz luz, então a luz UV deve vir da lanterna do smartphone. Isto será testado a seguir.

\section{Materiais e Métodos}

Fizemos medidas para obter o perfil de emissão das fontes de luz, usando um espectrofotômetro Ocean Optics USB 4000, região de trabalho de $300 \mathrm{~nm}$ a $1100 \mathrm{~nm}$. Nos gráficos mostrados posteriormente, suprimimos as regiões sem picos de emissão, para melhorar a visuali-

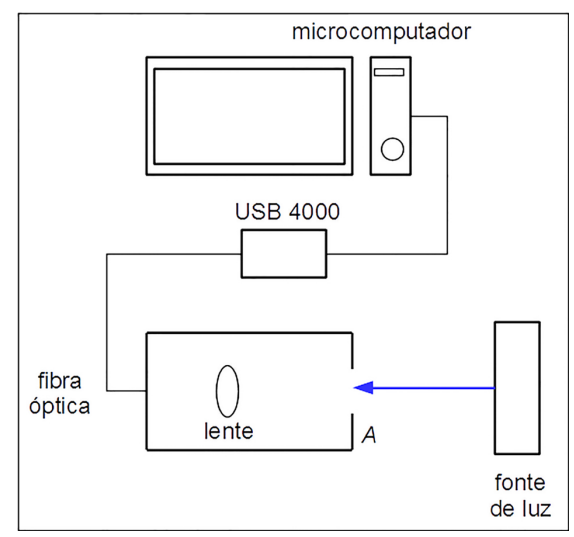

Figura 2: Esquema da montagem usada para obter o espectro de emissão.

zação dos picos de luz emitidos. Na Figura 2 vemos o arranjo experimental para medir os picos de emissão. A fonte de luz emite um feixe de luz, que entra em uma caixa, onde está acoplada uma fibra óptica e uma lente de vidro. A luz é concentrada pela lente na posição da ponta da fibra óptica. A fibra conduz a luz para dentro do espectrofotômetro USB 4000 e ele está conectado a um microcomputador. Um software proprietário da Ocean Optics controla o espectrofotômetro e fornece o perfil de emissão. Os dados são armazenados no microcomputador e processados posteriormente. Na posição $A$ foram colocados os filtros de luz, assim podemos filtrar a luz da fonte antes de entrar na caixa.

Para comparar, usamos uma lâmpada fluorescente de luz negra (Fig. 3a), cuja potência é de $28 \mathrm{~W}$ e bulbo de vidro.

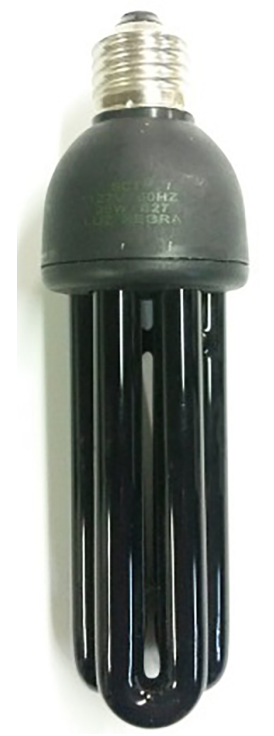

(a) Lâmpada de luz negra.

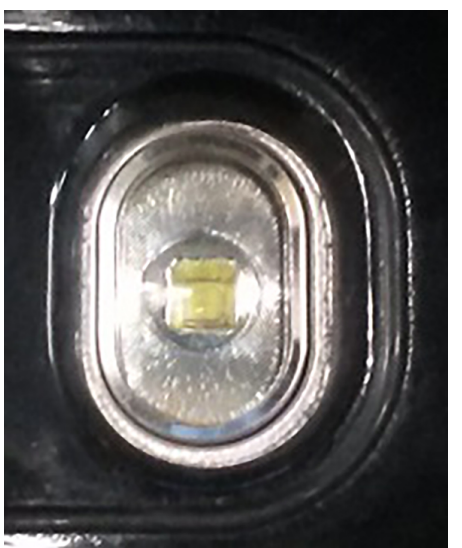

(b) Lanterna de smartphone.

Figura 3: Imagens da fontes de luz utilizadas. 
Foram estudadas as lanternas de três smartphones diferentes, sendo que a marca de um é diferente dos demais, mas todos são de modelos diferentes, identificados como J7, S4 e G3. Pretendemos verificar se algum deles emite luz UV. A lanterna de todos estes smartphones é formada por um LED. Na Figura 3b, vemos o LED do smartphone $\mathrm{J} 7$, que foi usado para iluminar as amostras com o filtro azul citado anteriormente.

Utilizamos filtros coloridos (azul e vermelho) e um filme prateado. Foi construido um filtro azul (Fig. 4a), de dimensões $(8 \times 5) \mathrm{cm}^{2}$, conforme descrito anteriormente, vide seção 2 O filtro vermelho (Fig. 4b), dimensões de (14 $\mathrm{x} 15) \mathrm{cm}^{2}$, era uma folha de papel celofane vermelho. $\mathrm{O}$ filme prateado (Fig $4 \mathrm{c}$ ), dimensões de $(21 \times 15) \mathrm{cm}^{2}$, era uma película de controle solar que é colada nos vidros de janelas, para barrar alguns comprimentos de onda da luz solar, visando melhorar o conforto dentro de edificações, mas que permite a passagem da luz visível para favorecer a iluminação natural do ambiente. Os filtros e o filme são importantes para analisar as luzes utilizadas neste trabalho bloqueando as luzes de alguns comprimentos de onda, como será visto na próxima seção.

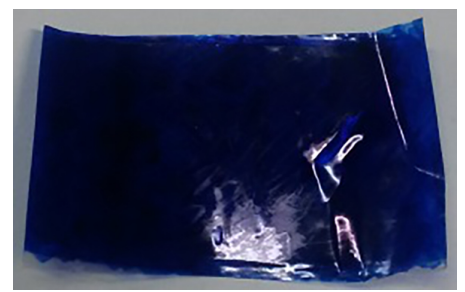

(a) Filtro Azul.

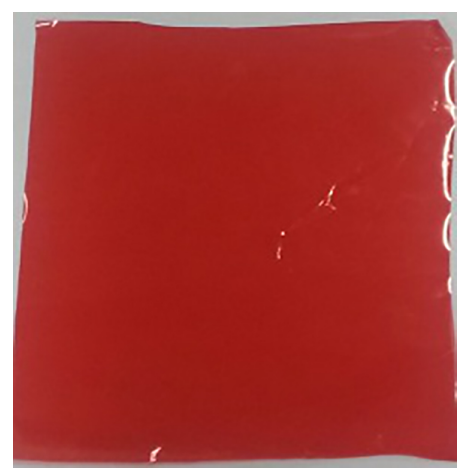

(b) Filtro Vermelho.

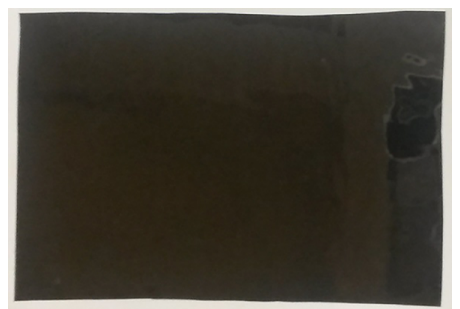

(c) Filme Prateado.

Figura 4: Imagens dos filtros utilizados nos experimentos.
Alguns cuidados devem ser tomados ao utilizar fontes de luz UV, no mínimo utilizar um óculos de proteção a radiação UV. Como citado anteriormente, a luz UV pode danificar os olhos. É aconselhável consultar o manual de cada fonte de luz UV, que será utilizada, e ler os cuidados de segurança a serem tomados. Utilizamos os óculos de segurança LPS-HeNe da Uves, este equipamento absorve luz UV e luz laser na região da cor vermelha.

Na Figura 5a vemos o esquema da montagem usada para registrar as fotografias da amostra iluminada pela fonte de luz. Esta amostra foi iluminada, tanto pela lanterna de smartphone, quanto pela lâmpada de luz negra. Também vemos que os filtros de luz são postos à frente da fonte.

A amostra utilizada era uma folha de papel de dimensões $(10,5 \times 10,5) \mathrm{cm}^{2}$, sendo que metade dessa folha foi pintada com caneta marca texto de cor verde e a outra metade com cor amarela. Iluminando com luz branca, vemos as cores verde e amarela (Fig. $5 \mathrm{~b}$ ).

As imagens foram registradas por uma câmera digital, que estava acima da amostra, deste modo foi possível registar a totalidade da amostra.

\section{Resultados}

Com o espectrofotômetro foram feitas 5 medidas de cada caso. Foram calculados os valores médios dessas medidas e depois foram normalizados.

Na Figura 6, vemos o gráfico da intensidade normalizada da lâmpada de luz negra (linha preta). Há apenas dois picos de emissão, o principal em 380 nm e o segundo em $410 \mathrm{~nm}$. Usamos um filtro vermelho de papel celofane (linha vermelha), para eliminar a emissão em $410 \mathrm{~nm}$, o que foi alcançado, mas com forte atenuação do pico em UV. Assim, podemos investigar a amostra iluminada com luz UV, sem nenhum componente na região do visível. Também usamos um filme prateado (linha verde escuro) para eliminar a emissão UV, podemos ver no gráfico que o filme barra os dois picos de emissão da lâmpada.

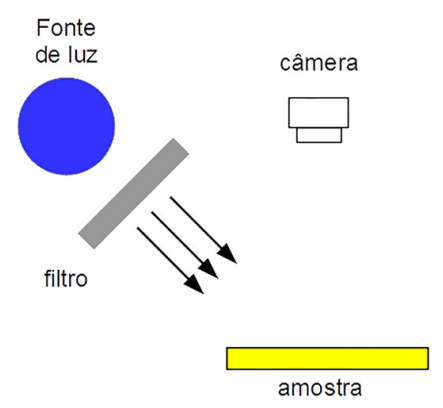

(a) Esquema da montagem.

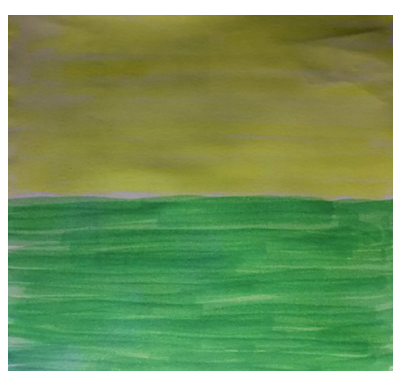

(b) Imagem da amostra iluminada por luz branca.
Figura 5: Esquema da montagem experimental para registar imagens da amostra iluminada. 


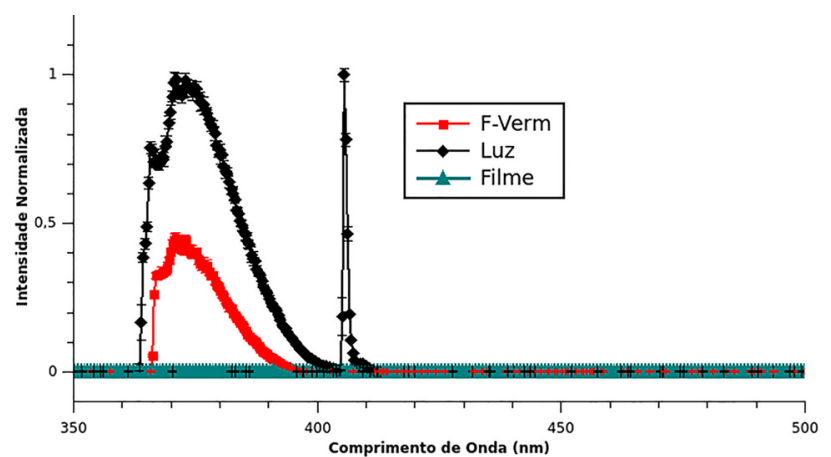

Figura 6: Gráfico da intensidade normalizada da lâmpada de luz negra, e usando um filtro vermelho e um filme prateado.

Na Figura 7, vemos a intensidade normalizada das três lanternas (J7, S4 e G3), em função do comprimento de onda, vemos que não há emissão de luz UV, e cada curva apresenta dois picos, um pico na região da cor azul (450 $\mathrm{nm})$ e o outro na região da cor verde $(550 \mathrm{~nm})$. Neste gráfico vemos que os três equipamentos não emitem luz UV (sem o filtro azul) então podemos extrapolar este comportamento para outros smartphones afirmando que não emitem luz UV, até que se prove o contrário.

$\mathrm{Na}$ Figura 8, vemos a intensidade normalizada da lanterna do smartphone J7 e a curva da mesma lanterna com o filtro azul, ele elimina o pico em $550 \mathrm{~nm}$ e atenua o pico em $450 \mathrm{~nm}$, não vemos nenhum pico de emissão na região da luz UV, logo a luz resultante deste sistema é da cor azul sem a presença de luz UV.

$\mathrm{Na}$ Figura 9a vemos a amostra fluorescendo sob a luz negra da lâmpada fluorescente com o filtro vermelho. Lembrando que o papel foi pintado com as cores verde e amarelo (vide Fig. 5b), as duas partes fluorescem luz verde. Como na luz utilizada não há componente da luz visível, não vemos diferenças entre elas. A frequência da luz emitida pela amostra é diferente da luz UV. A luz que fluoresce é da cor verde na região de $550 \mathrm{~nm}$ e a luz

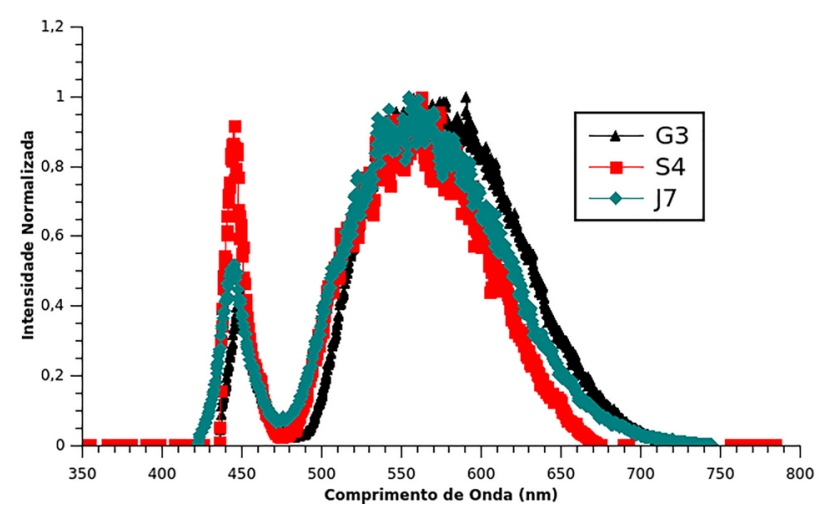

Figura 7: Gráfico da intensidade normalizada da emissão das três lanternas de smartphone (J7, S4 e G3) em função do comprimento de onda.

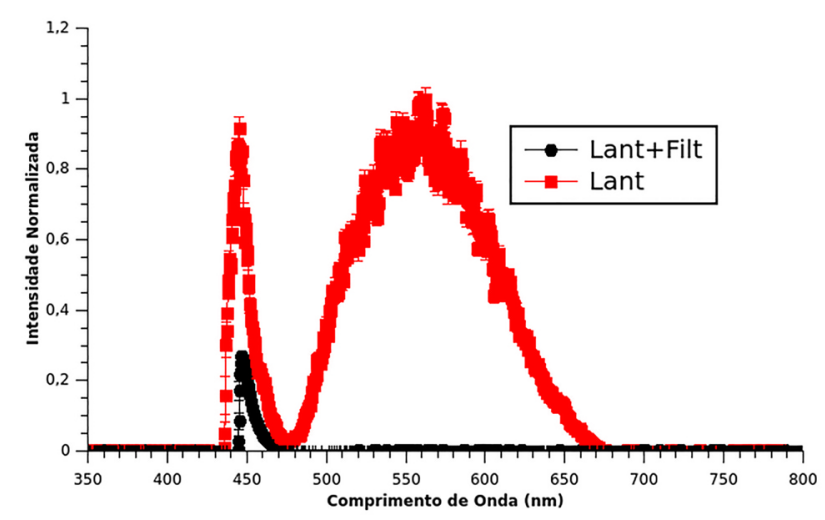

Figura 8: Gráfico da intensidade normalizada da emissão da lanterna do smartphone $\mathrm{J} 7$ em função do comprimento de onda, com (Lant+Filt) e sem o filtro azul (Lant).

UV utilizada está abaixo de $400 \mathrm{~nm}$. Ao adicionar o filme prateado, vide Figura 9b, não há fluorescência, pois o filme barra a luz UV, então não há luz visível vindo da amostra.

Na Figura 10a, vemos a amostra iluminada pela lanterna de smartphone J7 com o filtro azul. A amostra parece azulada em ambas as partes; não vemos semelhança com a Figura 9a. A luz advindo da amostra é da cor azul, parecida com a luz que foi emitida pelo sistema lanterna-filtro. Ao acrescentar o filme prateado para barrar luz UV (Fig. 10b), ocorreu uma atenuação da

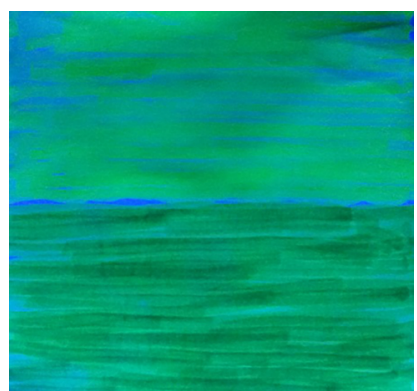

(a) Com o filtro vermelho.

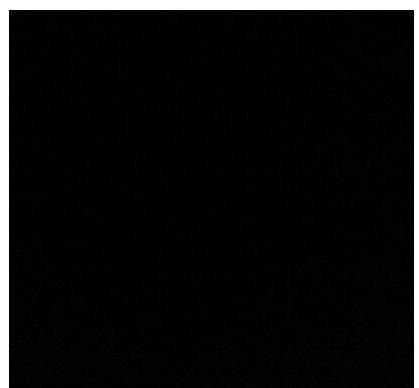

(b) Adicionado o filme prata.
Figura 9: Imagens da amostra iluminada pela lâmpada de luz negra.

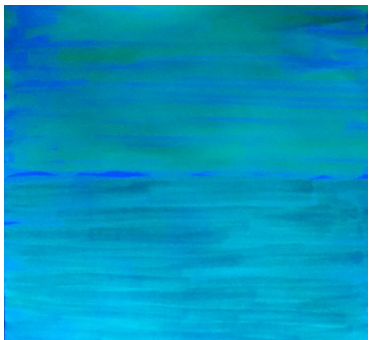

(a) Com o filtro azul.

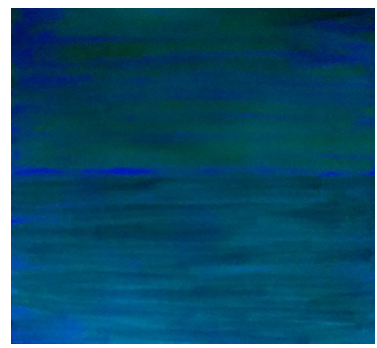

(b) Adicionado o filme prata.
Figura 10: Imagens da amostra iluminada pela lanterna de smartphone $\mathrm{J} 7$ com o filtro azul. 
intensidade, sem outras diferenças. Novamente a amostra parece azul. Neste caso, não vemos a mudança da frequência da luz advindo da amostra, em relação a luz que a ilumina. Portanto, podemos concluir que a proposta da lanterna de smartphone com filtro azul, não emite luz UV. Então, não é perigoso as pessoas utilizarem a lanterna do smartphone, porque ela não funciona como fonte de luz UV.

\section{Conclusão}

Neste trabalho foi estudada a proposta da lanterna de smartphone com filtro azul, para confirmar se, de fato, ela emite luz UV. Os resultados mostraram que o sistema não emite esse tipo de luz e que as pessoas podem manusear o equipamento, sem que este lhes cause danos. Pode-se afirmar também, que não é possível usar e/ou realizar experimentos de fluorescência com este tipo de fonte de luz. A pergunta feita na introdução foi respondida, a luz da lanterna de smartphone é segura.

\section{Referências}

[1] E. Hecht, Óptica. (Fundação Calouste Gulbenkian, Lisboa, 1991).

[2] A.L.P. Nery e C. Fernandez, Química Nova na Escola 19, 39 (2004).

[3] J.R. Pimentel, F.D. Saad, P. Yamamura, C.H. Furukawa, e V.H. Zumpano, Caderno Brasileiro de Ensino de Física 31, 365 (2014).

[4] W.L. Almeida, F.M.M. Luz, J.B. Silva, S.L.R. Silva, e A.M. Brinatti, Caderno Brasileiro Ensino de Física 30, 396 (2013).

[5] https://www.youtube.com/watch?v=AJt6PJlzVNQ, Acessadoem21/09/2018.

[6] https://www .youtube.com/watch?v=ymFMgmeqXOk, Acessadoem21/09/2018.

[7] https://www $\cdot$ youtube.com/watch?reload=9\&v= 0jrWqoHTS6c , Acessadoem22/03/2019. 